\title{
Neumotórax espontáneo bilateral en un paciente con distrofia muscular de Duchenne
}

\author{
Gildardo Cortés, * $₫$ José M Mier, * Luis C Valencia,* \\ Patricio Santillán-Doherty* \\ *Departamento de Cirugía, Instituto Nacional de Enfermedades Respiratorias Ismael Cosío Villegas, Ciudad de México; \\ †Departamento de Cirugía General, Centro Médico ABC, Ciudad de México. \\ Trabajo recibido: 19-VIII-2014; aceptado: 15-IX-2014
}

\begin{abstract}
RESUMEN. Introducción: Los pacientes con distrofia muscular de Duchenne comúnmente requieren de apoyo con ventilación no invasiva, lo cual incrementa el riesgo de neumotórax espontáneo secundario. Caso clínico: Presentamos el caso de un paciente masculino de 24 años de edad, con distrofia muscular de Duchenne y uso prolongado de ventilación no invasiva. Presentó neumotórax bilateral y sincrónico, manejado con pleurostomía y pleurodesis. Seis semanas después presentó recurrencia del neumotórax izquierdo, por lo que se llevó a cabo bulectomía por cirugía toracoscópica videoasistida y dos meses más tarde recurrió del lado derecho y se realizó mismo procedimiento. Evolucionó satisfactoriamente y no ha presentado recurrencia en un seguimiento a 18 meses. Discusión y conclusiones: La recurrencia del neumotórax espontáneo secundario se presenta en un $50 \%$ de los casos aproximadamente. Se recomienda el manejo quirúrgico posterior al primer episodio de neumotórax espontáneo, ya que la morbilidad y mortalidad es hasta cuatro veces mayor a partir del segundo episodio. El manejo toracoscópico en este paciente fue efectivo, seguro y bien tolerado. Se requieren más pacientes para darle consistencia a los hallazgos, así como de un estudio clínico comparativo de las terapias existentes para emitir una recomendación.
\end{abstract}

Palabras clave: Distrofia muscular de Duchenne, neumotórax espontáneo secundario, VATS.

\begin{abstract}
Background: Patients with Duchenne muscular dystrophy commonly needs non-invasive ventilation, which increases risk of secondary spontaneous pneumothorax. Clinical case: Male patient, 24 years old, with Duchenne muscular dystrophy and long term use of non-invasive ventilation. He presented bilateral and synchronic pneumothorax, managed with pleurostomy and bed pleurodesis. Nevertheless six weeks later he presented recurrence of left pneumothorax, so we performed bullectomy with video assisted thoracoscopic surgery and 8 weeks after this the right side also presented pneumothorax, giving the same manage. The patient recovered well and has not presented recurrence in eighteen month of follow up. Discussion and conclusions: Recurrence of secondary spontaneous pneumothorax occurs in approximately $50 \%$ of cases. After the first episode of spontaneous pneumothorax surgical management is recommended, because morbidity and mortality is up to 4 times higher in the second episode. In this case the surgical management with thoracoscopic was effective, secure and well tolerated. More patients are required to give consistency to the findings as well as a comparative clinical study of existing therapies to emit a recommendation.
\end{abstract}

Key words: Duchenne muscular dystrophy, secondary spontaneous pneumothorax, VATS.

\section{INTRODUCCIÓN}

La distrofia muscular de Duchenne (DMD) es la más comúnmente hereditaria. Estos pacientes mueren alrededor de la tercera década de la vida por falla respiratoria y neumonía. La mayor parte de los pacientes requiere el apoyo de ventilación no invasiva (VNI) después de 20 años, situación ésta que aumenta el riesgo de desarrollar neumotórax espontáneo secundario (NES). ${ }^{1}$

El tratamiento actual del NES consiste en administrar oxígeno suplementario, estabilización fisiológica, colocación de sonda endopleural (SEP) y el manejo de fugas aéreas persistentes. Se puede prevenir la recurrencia de NES utilizando pleurodesis química en pacientes que no sean candidatos a cirugía; así como cirugía abierta o cirugía toracoscópica videoasistida (VATS) en pacientes sin contraindicaciones. ${ }^{2}$

La fragilidad de los pacientes con DMD hace que comúnmente se evite el manejo quirúrgico; sin embargo, este grupo de pacientes se pueden beneficiar de VATS. ${ }^{3}$ 


\section{CASO CLÍNICO}

Paciente masculino de 24 años edad, con diagnóstico de DMD y antecedente de estabilización de columna a los 11 años de edad. Requirió de VNI con presión positiva de vía aérea de dos niveles (BIPAP) desde los 22 años.

Presentó cuadro de disnea de inicio súbito, ansiedad y mareo. Durante el abordaje se evidenció neumotórax bilateral y sincrónico, de $15 \%$ en el hemitórax derecho

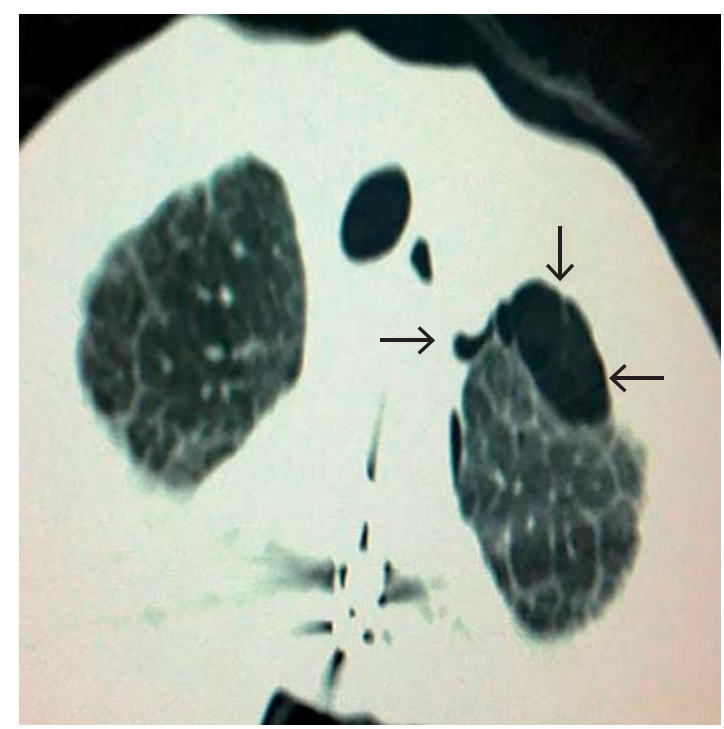

Figura 1. Tomografía computada de tórax con ventana para pulmón donde se observa bula apical (flechas) en el pulmón izquierdo. No se observan bulas en el pulmón derecho.

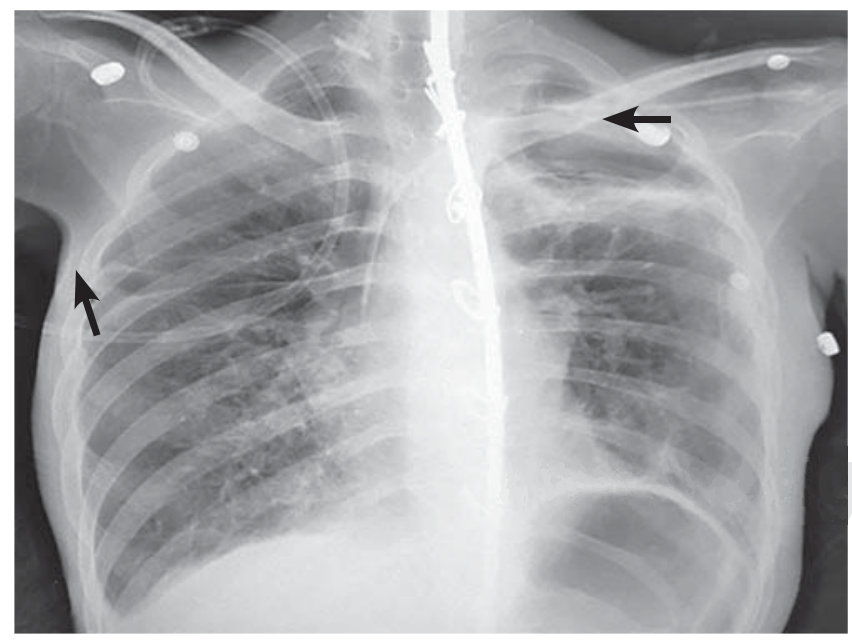

Figura 2. Radiografía de tórax tomada a los 12 días de la segunda cirugía, donde se observa cavidad apical residual al procedimiento previo en el hemitórax izquierdo, así como expansión total del pulmón derecho posterior al retiro del SEP. y de $50 \%$ en el izquierdo. De manera inicial fue manejado con colocación de SEP y pleurodesis del lado izquierdo nada más. La SEP se mantuvo por 15 días debido a una fuga aérea persistente. Seis semanas después, presentó sintomatología inicial y nuevamente le fue diagnosticado NES izquierdo del $50 \%$, situación para colocar SEP y realizar tomografía computada que documentó algunas bulas apicales bilaterales sin hallazgos relevantes en el resto del parénquima (figura 1). Se decide manejo quirúrgico.

Bajo anestesia general e intubación selectiva orotraqueal, se realizó VATS con dos puertos en hemitórax izquierdo, uno de $4 \mathrm{~cm}$ en el cuarto espacio intercostal y otro de $1 \mathrm{~cm}$ en el sexto espacio intercostal para la cámara de video. Se hizo adherenciolisis y bulectomía apical izquierda utilizando engrapadora endoscópica con cartuchos de $45 \mathrm{~mm}$ con grapas de $3.5 \mathrm{~mm}$, reforzando línea de grapas con sellador tisular de cianocrilato. Se llevó a cabo pleurodesis abrasiva y química con yodopovidona. Finalmente se colocó SEP calibre $24 \mathrm{Fr}$.

La evolución postoperatoria fue satisfactoria, se retiró la SEP a las 48 horas y egresó al tercer día postoperatorio. El estudio histopatológico reportó bulas tipo 1 y 2 , enfisema obstructivo, así como neumonía organizada. Dos meses después, el paciente acudió al servicio de Urgencias con NES derecho del $70 \%$. El manejo fue el mismo, con resección apical de bulas y pleurodesis. Egresó 12 días después por fuga aérea prolongada (figura 2).

En un seguimiento a 18 meses, el paciente ya no ha presentado recurrencias; aunque mantiene una cavidad pleural por falta de reexpansión total que se ha venido reduciendo con el tiempo.

\section{DISCUSIÓN Y CONCLUSIONES}

El término VNI hace referencia a dispositivos que proporcionan presión positiva continua de las vías respiratorias, BIPAP o insuflación-exuflación mecánica (MI-E) mejor conocido como Cough Assistant. ${ }^{2}$

La terapia con VNI ha incrementado la sobrevida de los pacientes con DMD aproximadamente 10 años más que los controles históricos. ${ }^{1}$ Lo anterior conlleva a un incremento de pacientes con falla respiratoria crónica que en forma inherente desarrollan complicaciones derivadas del uso de VNI. ${ }^{2-6}$ Por lo tanto, se ha vuelto una necesidad el plantear una estrategia de prevención, diagnóstico y tratamiento de las mismas.

La relación causal entre la VNI y NES es controversial. Existe la teoría de que la presión positiva daña el parénquima pulmonar e incrementa el riesgo de NES. Por otro lado, la asociación entre DMD y NES también es controversial. Se ha reportado un aumento en la 
incidencia de NES en pacientes con DMD en comparación con la población general. ${ }^{7}$ EI NES se desarrolla en pacientes con enfermedad clínica pulmonar, con una incidencia de 3.8 casos por 100,000 habitantes. ${ }^{8}$ En este caso, el paciente desarrolló neumonitis crónica y bulas apicales. Existe la teoría de una secuencia en la que la enfermedad causa debilidad para la ventilación, y el uso continuo de VNI provocan un daño inflamatorio del parénquima que finalmente desencadenan necrosis tisular y NES. ${ }^{8,9}$ Actualmente el conocimiento de la fisiopatología del daño parenquimatoso por la VNI nos ha llevado a no usar la MI-E en pacientes con antecedente de neumotórax o bulas. ${ }^{2}$

Las recurrencia del NES en la población general es de un $50 \%$ de los casos aproximadamente. Algunas sociedades de neumología recomiendan el manejo quirúrgico posterior al primer episodio de NES, ya que la morbilidad y mortalidad es hasta cuatro veces mayor a partir del segundo episodio. ${ }^{8}$ Yamamoto et al., reportaron $18 \%$ de NES en 50 pacientes con DMD, quienes no presentaron bulas, blebs o infiltrados por imagen; y en el seguimiento, 4 de 9 pacientes tuvieron

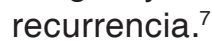

El tratamiento del NES en el contexto de DMD se ha descrito en diferentes formas. ${ }^{2,4,7,9} \mathrm{Se}$ ha optado por la terapia conservadora con SEP y pleurodesis, como lo recomiendan las guías de la Sociedad Española de Neumología y Cirugía Torácica y de la Sociedad Torácica Británica. 8,10 Un factor determinante de tal manejo es considerar a los pacientes con DMD como un grupo frágil, con un riesgo elevado de complicaciones para una cirugía torácica. Incluso las guías internacionales proponen la toracotomía axilar sobre VATS en cuanto a cuestiones de eficacia y probabilidad de recidiva. ${ }^{8}$ Nuestro grupo propone bulectomía y pleurodesis por VATS al extrapolar los buenos resultados, y la seguridad que otros autores y nosotros hemos observado en pacientes con neumotórax espontáneo primario y secundario, con efectividad del $96.1 \%$ de libertad de recurrencias de NES en un seguimiento a 10 años. ${ }^{11-14}$

Respecto a la técnica utilizamos engrapadoras con cartuchos de $3.5 \mathrm{~mm}$, lo que es adecuado para el parénquima pulmonar de características normales. Sin embargo, en el grupo de pacientes con patología como el caso descrito, es más adecuado el uso de cartuchos reforzados con una cintilla de pericardio o PTFE..$^{15}$ También se uso sellantes tisulares de cianocrilato, dada la evidencia aportada por otros estudios que recomiendan su uso en la prevención de la fuga aérea en el contexto de cirugía de bulas o enfisema. ${ }^{16}$

En el caso presentado, el manejo quirúrgico con VATS fue efectivo, seguro y bien tolerado. Se requieren más pacientes para darle consistencia a los hallazgos, así como de un estudio clínico comparativo de las terapias existentes para emitir una recomendación.

\section{REFERENCIAS}

1. Kohler M, Clarenbach CF, Bahler C, Brack T, Russi EW, Bloch KE. Disability and survival in Duchenne muscular dystrophy. J Neurol Neurosurg Psychiatry 2009;80(3):320-325. doi: 10.1136/jnnp.2007.141721.

2. Suri P, Burns SP, Bach JR. Pneumothorax associated with mechanical insufflation-exsufflation and related factors. Am J Phys Med Rehabil 2008;87(11):951-955. doi: 10.1097/PHM.0b013e31817c181e.

3. Kang SW, Bach JR. Maximum insufflation capacity: vital capacity and cough flows in neuromuscular disease. Am J Phys Med Rehabil 2000;79(3):222-227.

4. Vianello A, Arcaro G, Gallan F, Ori C, Bevilacqua M. Pneumothorax associated with long-term non-invasive positive pressure ventilation in Duchenne muscular dystrophy. Neuromuscul Disord 2004;14(6):353-355.

5. Choo-Kang LR, Ogunlesi FO, McGrath-Morrow SA, Crawford TO, Marcus CL. Recurrent pneumothoraces associated with nocturnal noninvasive ventilation in a patient with muscular dystrophy. Pediatr Pulmonol 2002;34(1):73-78.

6. Salazar-Otaola GF, Ibarra-Celaya JM, Vázquez-Minero JC. Ruptura esofágica: presentación de un problema inusual. Reporte de un caso. Neumol Cir Torax 2013;72(3):218-222.

7. Yamamoto T, Kawai M. Spontaneous pneumothorax in Duchenne muscular dystrophy. Rinsho Shinkeigaku 1994;34(6):552-556.

8. Rivas de Andrés JJ, Jiménez LMF, Molins LL, Pérez TA, Torres LJ. Normativa sobre el diagnóstico y tratamiento del neumotórax espontáneo. Arch Bronconeumol 2008;44(8):437-448.

9. Sahn SA, Heffner JE. Spontaneous pneumothorax. N Engl J Med 2000;342(12):868-874.

10. MacDuff A, Arnold A, Harvey J; BTS Pleural Disease Guideline Group. Management of spontaneous pneumothorax: British Thoracic Society Pleural Disease Guideline 2010. Thorax 2010;65(Suppl 2):ii18-31. doi: 10.1136/thx.2010.136986.

11. Santillán-Doherty P, Argote-Greene LM, Guzman-Sanchez M. Thoracoscopic management of primary spontaneous pneumothorax. Am Surg 2006;72(2):145-149.

12. Shaikhrezai K, Thompson Al, Parkin C, Stamenkovic S, Walker WS. Video-assisted thoracoscopic surgery management of spontaneous pneumothorax--long-term results. Eur J Cardiothorac Surg 2011;40(1):120-123. doi: 10.1016/j.ejcts.2010.10.012.

13. Kim SJ, Lee HS, Kim HS, et al. Outcome of video-assisted thoracoscopic surgery for spontaneous secondary pneumothorax. Korean J Thorac Cardiovasc Surg 2011;44(3):225-228. doi: 10.5090/kjtcs.2011.44.3.225.

14. Zeybek $A$, Kalemci $S$, Gürünlü $O$, Süzen $A$, Akgül $M$, Koç K. The effect of additional pleural procedures onto 
recurrence rates on the spontaneous pneumothorax surgery. Iran Red Crescent Med J 2013;15(2):136-141. doi: 10.5812/ircmj.7990.

15. Murray KD, Ho CH, Hsia JY, Little AG. The influence of pulmonary staple line reinforcement on air leaks. Chest 2002;122(6):2146-2149.

16. Carr JA. The intracorporeal use of 2-octyl cyanoacrylate resin to control air leaks after lung resection. Eur J Cardiothorac Surg 2011;39(4):579-583. doi: 10.1016/j. ejcts.2010.07.036. $\triangle$ Correspondencia:

Dr. Gildardo Cortés Julián,

Departamento de Cirugía,

Instituto Nacional de Enfermedades

Respiratorias Ismael Cosío Villegas.

Calzada de Tlalpan Núm. 4502,

Colonia Sección XVI, México, D.F., 14080.

Teléfono: 54871700 extensión 5210, fax: 56660997

Correo electrónico: gildardounam@hotmail.com

Los autores declaran no tener conflicto de intereses 